\title{
Dirichlet problems for linear and semilinear sub- Laplace equations on Carnot groups
}

Zixia Yuan $^{1 *}$ and Guanxiu Yuan ${ }^{2}$

* Correspondence: yzx8047@yahoo. com.cn

'School of Mathematical Science, University of Electronic Science and Technology of China,

Chengdu 611731, China

Full list of author information is available at the end of the article

\begin{abstract}
The purpose of this article, is to study the Dirichlet problems of the sub-Laplace equation $L u+f(\xi, u)=0$, where $L$ is the sub-Laplacian on the Carnot group $G$ and $f$ is a smooth function. By extending the Perron method in the Euclidean space to the Carnot group and constructing barrier functions, we establish the existence and uniqueness of solutions for the linear Dirichlet problems under certain conditions on the domains. Furthermore, the solvability of semilinear Dirichlet problems is proved via the previous results and the monotone iteration scheme corresponding to the sub-Laplacian.

Mathematics Subject Classifications: 35J25, 35J70, 35J60.
\end{abstract}

Keywords: Carnot group, sub-Laplace equation, Dirichlet problem, Perron method, monotone iteration scheme

\section{Introduction}

In this article we consider Dirichlet problems of the type

$$
\begin{cases}L u+f(\xi, u)=0, & \text { in } \Omega, \\ u=\varphi, & \text { on } \partial \Omega,\end{cases}
$$

where $\Omega$ is a bounded domain in a Carnot group $G$ and $L$ is the sub-Laplacian. Some knowledge on $G$ and $L$ see next section. Hörmander's theorem permits us to judge the hypoellipticity of the operator $L$, i.e., if $L u \in C^{\infty}$ then $u \in C^{\infty}$ (see [1]).

The investigation of the boundary value problems, concerning the operators in the form of the sum of squares of vector fields fulfilling Hörmander condition, has turned into the subject of several works, see [2-4]. The precursory work of Bony [2] proved a maximum principle and the solvability of the Dirichlet problem in the sense of PerronWiener. The Wiener type regularity of boundary points for the Dirichlet problem was considered in [3]. Thanks to the previous results, Capogna et al. [4] established the solvability of the Dirichlet problem when the boundary datum belongs to $L^{p}, 1<p \leq \infty$, in the group of Heisenberg type.

The Perron method (see $[5,6])$ and the monotone iteration scheme (see $[7,8])$ are well-known constructive methods for solving linear and semilinear Dirichlet problems, respectively. Brandolini et al. [9] applied these methods to the Dirichlet problems for sub-Laplace equations on the gauge balls in the Heisenberg group which is the simplest Carnot group of step two. Let us notice that the balls possess of legible properties. However, we do not see the reseach to the problems on other domains using

(C) 2012 Yuan and Yuan; licensee Springer. This is an Open Access article distributed under the terms of the Creative Commons Attribution License (http://creativecommons.org/licenses/by/2.0), which permits unrestricted use, distribution, and reproduction in any medium, provided the original work is properly cited. 
these methods. Concerning the construction of barrier function, Brandolini et al. [9] used the result given in [10], which holds in the setting of Heisenberg group.

Our work is motivated by [9]. We try to extend the existence of solutions for semilinear Dirichlet problems on the Heisenberg balls in [9] to general Carnot domains. To do so, the Perron method in the Carnot group is used in this article. Based on the work in [3], we construct a barrier function in a domain of the Carnot group (see Lemma 3.10) under the hypothesis of the outer sphere condition to discuss the boundary behaviour of the Perron solutions. The method to obtain a barrier function is essentially similar to the one in [9]. Then we prove the existence of solutions for linear sub-Laplace Dirichlet problems. In the discussion of semilinear Dirichlet problems, we will use monotone iteration scheme. The main difficulty we meet is that the sub-Laplacian $L$ does not have explicit expression. To overcome it, we use the regularity of $L$ in [1].

The article is organized as follows. In the next section, we recall some basic definitions and collect some known results on the Carnot group which will play a role in the following sections. Section 3 is devoted to the study of the Perron method for linear equations. By finding a barrier function related to the sub-Laplacian $L$, we prove that the Perron solutions for linear Dirichlet problems are continuous up to the boundary. The main results are Theorems 3.8, 3.11, and 3.13. In Section 4, using the results in Section 3 and the monotone iteration scheme, we provide the solutions of the semilinear Dirichlet problems in Carnot groups with some available supersolutions and subsolutions. Finally, we give an existence of solution to the sub-Laplace equation on the whole group of Heisenberg type (a specific Carnot group of step two). The main results in this section are Theorems 4.2 and 4.3.

\section{Carnot groups}

We will consider $G=\left(\mathbb{R}^{N}, \cdot\right)$ as a Carnot group with a group operation $\cdot$ and a family of dilations, compatible with the Lie structure.

Following [11,12], a Carnot group $G$ of step $r \geq 1$ is a simple connected nilpotent Lie group whose Lie algebra $\tilde{g}$ admits a stratification. That is, there exist linear subspaces $V_{1}, \ldots, V_{r}$ of $\tilde{g}$ such that

$$
\tilde{g}=\oplus_{j=1}^{r} V_{j}, \quad\left[V_{1}, V_{i}\right]=V_{i+1} \text { for } i=1,2, \ldots, r-1 \text { and }\left[V_{1}, V_{r}\right]=\{0\} .
$$

Via the exponential map, it is possible to induce on $G$ a family of non-isotropic dilations defined by

$$
\delta_{\lambda}(\xi)=\delta_{\lambda}\left(x^{(1)}, x^{(2)}, \ldots, x^{(r)}\right)=\left(\lambda x^{(1)}, \lambda^{2} x^{(2)}, \ldots, \lambda^{r} x^{(r)}\right) .
$$

Here $\xi=\left(x^{(1)}, x^{(2)}, \ldots, x^{(r)}\right), x^{(i)} \in \mathbb{R}^{N_{i}}$ for $i=1, \ldots, r$ and $N_{1}+\cdots+N_{r}=N$. We denote by $Q=\sum_{j=1}^{r} j N_{j}$ the homogeneous dimension of $G$ attached to the dilations $\left\{\delta_{\lambda}\right\}$ $\lambda>0$. Let $m=N_{1}$ and $X=\left\{X_{1}, \ldots, X_{m}\right\}$ be the dimension and a basis of $V_{1}$, respectively. Let $X u=\left\{X_{1} u, \ldots, X_{m} u\right\}$ denote the horizontal gradient for a function $u$. The sub-Laplacian associated with $X$ on $G$ is given by

$$
L=\sum_{j=1}^{m} X_{j}^{2} .
$$


If $u$ and $v$ are two measurable functions on $G$, their convolution is defined by

$$
u * v(\xi)=\int_{G} u(\eta) v\left(\eta^{-1} \cdot \xi\right) d G(\eta)=\int_{G} u\left(\xi \cdot \eta^{-1}\right) v(\eta) d G(\eta),
$$

where $d G(\eta)$ denotes a fixed Haar measure on $G$.

Let $e$ be the identity on $G$. For $\xi \in G$, we denote by $\xi^{-1}$ the inverse of $\xi$ with respect to the group operation. By [1], there exists a norm function $\rho(\xi) \in C_{0}^{\infty}(G \backslash\{e\}) \cap C(G)$ satisfying

(1) $\rho(\xi) \geq 0$; Moreover, $\rho(\xi)=0$ if and only if $\xi=e$;

(2) $\rho(\xi)=\rho\left(\xi^{-1}\right)$.

The open ball of radius $R$ centered at $\xi$ is expressed as the set:

$$
B_{G}(\xi, R)=\left\{\eta \in G: \rho(\xi, \eta)=\rho\left(\xi^{-1} \cdot \eta\right)<R\right\} .
$$

Let $\mathcal{D}^{\prime}$ denote the space of distributions on $G$. The non-isotropic Sobolev space $S^{k, p}$ is defined by

$$
S^{k, p}=\left\{f \in \mathcal{D}^{\prime}: D^{\alpha} f \in L^{p}(G),|\alpha| \leq k\right\},
$$

where $\alpha=\left(\alpha_{1}, \ldots ., \alpha_{l}\right)$ is a multi-index, $D^{\alpha}=D_{\alpha_{1}} D_{\alpha_{2}} \cdots D_{\alpha_{l}}$, and $D_{\alpha_{j}} \in\left\{X_{1}, \ldots, X_{m}\right\}$. In the space $S^{k, p}$, we shall adopt the norm

$$
\|f\|_{S^{k, p}}=\sup _{|\alpha| \leq k}\left\|D^{\alpha} f\right\|_{L^{p}} .
$$

For a domain $\Omega$ in $G$, we define $S^{k, p}(\Omega, l o c)$ as the space of distributions $f$ such that for every $\psi(\xi) \in C_{0}^{\infty}(\Omega)$ we have $f \psi \in S^{k, p}$. Let $0<\beta<\infty$, we employ the following non-isotropic Lipschitz spaces:

(i) for $0<\beta<1$,

$$
\Gamma^{\beta}:=\left\{f \in L^{\infty} \cap C^{0}: \sup _{\xi, \eta} \frac{|f(\eta \cdot \xi)-f(\eta)|}{(\rho(\xi, e))^{\beta}}<\infty\right\},
$$

(ii) for $\beta=1$,

$$
\Gamma^{1}:=\left\{f \in L^{\infty} \cap C^{0}: \sup _{\xi, \eta} \frac{\left|f(\eta \cdot \xi)+f\left(\eta \cdot \xi^{-1}\right)-2 f(\eta)\right|}{(\rho(\xi, e))^{\beta}}<\infty\right\},
$$

(iii) for $\beta=k+\beta^{\prime}$ where $k=1,2,3, \ldots$ and, $0<\beta^{\prime} \leq 1$,

$$
\Gamma^{\beta}:=\left\{f \in L^{\infty} \cap C^{0}: D^{\alpha} f \in \Gamma^{\beta^{\prime}},|\alpha| \leq k\right\} .
$$

We refer the reader to [1] for more information on the above.

The following results are useful.

Proposition 2.1. (i) Suppose $\Omega \subset G$ is an open set, and suppose $f, g \in \mathcal{D}^{\prime}(\Omega)$ satisfy $L f=g$ in $\Omega$. If $g \in S^{k, p}(\Omega, l o c)(1<p<\infty, k \geq 0)$ then $f \in S^{k+2, p}(\Omega, l o c)$. 
(ii) Suppose $1<p<\infty$ and $\beta=k-\frac{Q}{p}>0$, then $S^{k, p} \subset \Gamma^{\beta}$.

Part (i) and (ii) are contained, respectively, in Theorems 6.1 and 5.15 of [1].

\section{The Perron method and barrier function for linear problem}

In this section, we study the solvability of the following linear sub-Laplace Dirichlet problem

$$
\begin{cases}L u-\lambda(\xi) u=f, & \text { in } \Omega, \\ u=\varphi, & \text { on } \partial \Omega,\end{cases}
$$

where $\lambda(\xi) \in C(\bar{\Omega})$ satisfies $\lambda(\xi)>0$.

Definition 3.1. A bounded open set $\Omega \subset G$ is said to satisfy the outer sphere condition at $\xi_{0} \in \partial \Omega$, if there exists a ball $B_{G}(\eta, r)$ lying in $G \backslash \Omega$ such that

$$
\partial B_{G}(\eta, r) \cap \partial \Omega=\left\{\xi_{0}\right\}
$$

The definition in the case of general degenerate elliptic operator can be seen in [3]. Notice that in the H-type group case, every bounded convex subset accords with the condition of the outer sphere. In particular, the gauge balls in H-type group are convex domains (see [4]). From Theorem 2.12 in [13] and Theorem 5.2 in [2] respectively, one has the following two lemmas.

Lemma 3.2. (Maximum principle) Let $\Omega$ be a connected open set in a Carnot group G. If $u \in C^{2}(\Omega)$ satisfies

$$
L u-\lambda(\xi) u \geq 0 \text { in } \Omega,
$$

then $u$ cannot achieve a nonnegative maximum at an interior point unless $u \equiv$ constant in $\Omega$.

Lemma 3.3. Let $\Omega$ be a bounded domain in G. Then there exists a family of open subsets, denoted by $\mathcal{F}=\{\omega: \bar{\omega} \subset \Omega\}$, which is a base for the topology of $\Omega$ for which the Dirichlet problem

$$
\begin{cases}L u-\lambda(\xi) u=f, & \text { in } \omega, \\ u=\varphi, & \text { on } \partial \omega\end{cases}
$$

has a unique distributional solution $u \in C(\bar{\omega})$ for any $\omega \in \mathcal{F}, f \in C(\bar{\omega})$ and $\phi \in C$ $(\partial \omega)$. Furthermore, if $f \in C^{\infty}(\omega)$, then $u \in C^{\infty}(\omega)$.

We give notions of subsolution and supersolution for the Dirichlet problem (3.1).

Definition 3.4. Let $\phi \in C(\partial \Omega), f \in C^{\infty}(\bar{\Omega})$. A function $u \in C(\bar{\Omega})$ is called a subsolution of (3.1) if it fits the following properties:

(i) $u \leq \phi$ on $\partial \Omega$;

(ii) for every $\omega \in \mathcal{F}$ and for every $h \in C^{2}(\omega) \cap C(\bar{\omega})$ such that $L h-\lambda(\xi) h=f$ and $u \leq h$ on $\partial \omega$, we also have $u \leq h$ in $\omega$.

The definition of supersolution is analogous.

Lemma 3.5. Assume that $u$ is a subsolution of (3.1) and $v$ is a supersolution of (3.1), then either $u<v$ in $\Omega$ or $u \equiv v$. 
Proof. Suppose that at some point $\eta \in \Omega$ we have $u(\eta) \geq v(\eta)$. Set $M=\sup _{\xi \in \Omega}(u-v)(\xi) \geq 0$. Take $\xi_{0} \in \Omega$ such that $(u-v)\left(\xi_{0}\right)=M$, and we can know that $u-v \equiv M$ in a neighborhood of $\xi_{0}$. Otherwise there exists $\omega \in \mathcal{F}$ such that $\xi_{0} \in \omega$ but $u-v$ is not identically equal to $M$ on $\partial \omega$. Letting $\bar{u}$ and $\bar{v}$ denote the solutions of $L w-\lambda(\xi) w=f$ in $\omega$, equal to $u$ and $v$ on $\partial \omega$ respectively. Since $u$ and $v$ are the subsolution and the supersolution respectively, we deduce from Definition 3.4 that $\bar{u} \geq u$ and $\bar{v} \leq v$ in $\omega$. One sees that

$$
M=\sup _{\xi \in \Omega}(u-v)(\xi) \geq \sup _{\xi \in \partial \omega}(\bar{u}-\bar{v})(\xi) \geq(\bar{u}-\bar{v})\left(\xi_{0}\right) \geq(u-v)\left(\xi_{0}\right)=M,
$$

and hence all the equalities above hold. By Lemma 3.2 it follows that $\bar{u}-\bar{v} \equiv M$ in $\omega$ and hence $u-v \equiv M$ on $\partial \omega$, which contradicts the choice of $\omega$.

The previous argument implies $u-v \equiv M$ in $\Omega$. Combining this with Definition 3.4-(i) we obtain $u \equiv v$ in $\Omega$.

Let $u \in C(\bar{\Omega})$ be a subsolution of (3.1) and $\omega \in \mathcal{F}$. Denote by $\bar{u}$ the solution of the Dirichlet problem (see Lemma 3.3)

$$
\begin{cases}L \bar{u}-\lambda(\xi) \bar{u}=f(\xi), & \text { in } \omega, \\ \bar{u}=u, & \text { on } \partial \omega,\end{cases}
$$

and define in $\Omega$ the lifting of $u$ (in $\omega$ ) by

$$
U(\xi):=\left\{\begin{array}{l}
\bar{u}(\xi), \quad \xi \in \omega, \\
u(\xi), \quad \xi \in \Omega \backslash \omega .
\end{array}\right.
$$

Lemma 3.6. $U(\xi)$ is a subsolution of (3.1).

Proof. Since $u(\xi)$ is a subsolution of (3.1), it follows that $U(\xi)=u(\xi) \leq \phi(\xi)$ on $\partial \Omega$. Let $\omega^{\prime} \in \mathcal{F}$ and $h \in C^{2}\left(\omega^{\prime}\right) \cap C\left(\overline{\omega^{\prime}}\right)$ such that $L h-\lambda(\xi) h=f$ and $U \leq h$ on $\partial \omega^{\prime}$. If $\omega \cap$ $\omega^{\prime}=\varphi$, then $u=U \leq h$ on $\partial \omega^{\prime}$. It leads to $U=u \leq h$ in $\omega^{\prime}$;

Suppose now $\omega \cap \omega^{\prime}=\varphi$. Since $u \leq U$, we have $u \leq h$ on $\partial \omega^{\prime}$ and then $u \leq h$ in $\omega^{\prime}$. In particular, $u \leq h$ in $\omega^{\prime} \mid \omega$, i.e. $U \leq h$ in $\omega^{\prime} \mid \omega$. Thus, we have $\bar{u} \leq h$ on $\partial\left(\omega^{\prime} \cap \omega\right)$. As $L(\bar{u}-h)-\lambda(\xi)(\bar{u}-h)=0$ in $\omega^{\prime} \cap \omega$ and $\bar{u}-h \leq 0$ on $\partial\left(\omega^{\prime} \cap \omega\right)$, it yields by Lemma 3.2 that $\bar{u} \leq h$ in $\omega^{\prime} \cap \omega$, and therefore $U \leq h$ in $\omega^{\prime} \cap \omega$.

The following result is a trivial consequence of Definition 3.4.

Lemma 3.7. Let $u_{1}, u_{2}, \ldots, u_{l}$ be subsolutions of (3.1). Then the function

$$
v=\max \left\{u_{1}, u_{2}, \ldots, u_{l}\right\}
$$

is also a subsolution of (3.1).

Let $S$ denote the set of all subsolutions of (3.1). Notice that $S$ is not empty, since $-k^{2} \in S$ for $k$ large enough. The basic result via the Perron method is contained in the following theorem.

Theorem 3.8. The function $u(\xi):=\sup _{v \in S} v(\xi)$ satisfies $L u-\lambda(\xi) u=f$ in $\Omega$.

Proof. Notice that $k^{2}$, for $k$ large enough, is a supersolution of (3.1). By Lemma 3.5, we deduce $v \leq k^{2}$ for any $v \in S$, so $u$ is well defined. Let $\eta$ be an arbitrary fixed point of $\Omega$. By the definition of $u$, there exists a sequence $\left\{v_{n}\right\}_{n \in \mathbb{N}}$ such that $v_{n}(\eta) \rightarrow u(\eta)$. By replacing $v_{n}$ with $\max \left\{v_{1}, \ldots, v_{n}\right\}$, we may assume that $v_{1} \leq v_{2} \leq \cdots \leq v_{n} \leq \cdots$. Let 
$\omega \in \mathcal{F}$ be such that $\eta \in \omega$ and define $V_{n}(\eta)$ to be the lifting of $v_{n}$ in $\omega$ according to (3.3). From Lemma 3.2, $V_{n}$ is also increasing and, since $V_{n} \in S$ (see Lemma 3.6) and $V_{n} \geq v_{n}$, it gets $V_{n}(\eta) \rightarrow u(\eta)$. Set $V(\xi):=\lim _{n \rightarrow \infty} V_{n}(\xi)$. Obviously, we have that $V \leq u$ in $\Omega$ and $V(\eta)=u(\eta)$. Noting that every $V_{n}$ satisfies $L V_{n}-\lambda(\xi) V_{n}=f$ in $\omega$, we have, by the dominated convergence theorem that the function $V$ satisfies $L V-\lambda(\xi) V=f$ in the distributional sense in $\omega$. Since $f \in C^{\infty}(\omega)$, we have $V(\xi) \in C^{\infty}(\omega)$ in view of the hypoellipticity of the operator $L-\lambda(\xi)$.

We conclude that $V \equiv u$ in $\omega$. In fact, suppose $V(\zeta)<u(\zeta)$ for some $\zeta \in \omega$, then there exists a function $\bar{u} \in S$ such that $V(\zeta)<\bar{u}(\zeta)$. Define the increasing sequence $w_{n}=\max \left\{\bar{u}, V_{n}\right\}$ and then the corresponding liftings $W_{n}$. Set $W(\xi):=\lim _{n \rightarrow \infty} W_{n}(\xi)$. Analogously to $V, W$ satisfies $L W-\lambda(\xi) W=f$. Since $V_{n} \leq w_{n} \leq W_{n}$, we obtain $V \leq W$. The equalities $V(\eta)=u(\eta)=W(\eta)$ and Lemma 3.2 imply that $V \equiv W$ in $\Omega$. This is in contradiction with $V(\zeta)<\bar{u}(\zeta) \leq W(\zeta)$. Consequently, $V \equiv u$ in $\omega$ and $u$ satisfies $L u$ $-\lambda(\xi) u=f$ in the classical sense. The arbitrariness of $\eta$ leads to the desired result. $\square$

Definition 3.9. Let $\zeta \in \partial \Omega$. Then a function $w(\xi) \in C^{\infty}(\Omega) \cap C(\bar{\Omega})$ is called a barrier function related to the sub-Laplacian $L$ at $\zeta$ if the following two conditions hold:

(i) $L w(\xi) \leq-1$ in $\Omega$;

(ii) $w(\xi)>0$ on $\bar{\Omega} \backslash\{\zeta\}, w(\zeta)=0$.

Lemma 3.10. Let $\Omega \subset G$ be a bounded open domain which satisfies the outer sphere condition at every point of the boundary $\partial \Omega$. Then for every $\zeta \in \partial \Omega$, the Dirichlet problem

$$
\begin{cases}L w=-1, & \text { in } \Omega \\ w(\xi)=\rho(\xi, \zeta), & \text { on } \partial \Omega\end{cases}
$$

has a unique solution $w \in C^{\infty}(\Omega) \cap C(\bar{\Omega})$ fulfilling $w(\zeta)>0$ on $\bar{\Omega} \backslash\{\zeta\}$ and $w(\zeta)=0$. Proof. From [1], let $\Gamma(\xi)=C_{\mathrm{Q}} \rho(\xi, e)^{-(\mathrm{Q}-2)}$ be the fundamental solution of the subLaplacian $L$. Define the convolution

$$
\tilde{u}:=-\Gamma * \chi_{\Omega},
$$

where $\chi_{\Omega}$ denotes the indicator function. Since $\Gamma(\xi) \in L_{l o c}^{p}$ for $1 \leq p<\frac{Q}{Q-2}$, it yields $\tilde{u} \in C^{\infty}(\Omega) \cap C(\bar{\Omega})$.

According to Corollary 10 in [3], the problem

$$
\begin{cases}L v=0, & \text { in } \Omega, \\ v(\xi)=\rho(\xi, \zeta)-\tilde{u}(\xi), & \text { on } \partial \Omega\end{cases}
$$

has a unique solution $v \in C^{\infty}(\Omega) \cap C(\bar{\Omega})$. Since $L \tilde{u}=-\chi_{\Omega}$ (see Corollary 2.8 in [1]), it follows that $w:=v+\tilde{u}$ is the desired solution of (3.4).

Theorem 3.11. Let $\Omega$ be as in Lemma 3.10. Suppose $\phi \in C(\partial \Omega)$ and $f \in C^{\infty}(\Omega) \cap C(\bar{\Omega})$. Then the Dirichlet problem (3.1) possesses a unique solution $u \in C^{\infty}(\Omega) \cap C(\bar{\Omega})$. 
Proof. Uniqueness is a direct consequence of Lemma 3.2. Theorem 3.8 provides the existence of the solution $u \in C^{\infty}(\Omega)$. To complete the proof of the theorem, it needs only to examine that $u$ is continuous up to the boundary of $\Omega$.

Let $\zeta \in \partial \Omega$. Since $\phi \in C(\partial \Omega)$, it follows that for any $\varepsilon>0$ there exists some $\delta>0$ such that for every $\xi \in \partial \Omega$ with $\rho(\xi, \zeta)<\delta$, we have

$$
|\varphi(\xi)-\varphi(\zeta)|<\varepsilon
$$

Let $w(\xi)$ be the barrier function related to $L$ at $\zeta$ constructed in Lemma 3.10. Set $M=\sup _{\xi \in \partial \Omega}|\varphi(\xi)|$ and choose $k_{1}>0$ such that $k_{1} w(\xi) \geq 2 M$ if $\rho(\xi, \zeta) \geq \delta$. Set $k_{2}=[|\varphi(\zeta)|+\varepsilon] \max _{\xi \in \Omega} \lambda(\xi)+\sup _{\xi \in \Omega}|f(\xi)|$, and $k=\max \left\{k_{1}, k_{2}\right\}$. Define that $w_{1}(\zeta):=\phi(\zeta)+$ $\varepsilon+k w(\xi)$ and $w_{2}(\xi):=\phi(\zeta)-\varepsilon-k w(\xi)$. Then we see in view of Lemma 3.10,

$$
L w_{1}-\lambda(\xi) w_{1}=-k-\lambda(\xi) \varphi(\zeta)-\lambda(\xi) \varepsilon-k \lambda(\xi) w(\xi) \leq f \quad \text { in } \Omega .
$$

On the one hand, $w_{1}(\xi)=\phi(\zeta)+\varepsilon+k w(\xi) \geq \phi(\zeta)+\varepsilon>\phi(\xi)$ when $\rho(\xi, \zeta)<\delta$; On the other hand, $w_{1}(\xi) \geq \phi(\zeta)+\varepsilon+2 M>\phi(\xi)$ when $\rho(\xi, \zeta) \geq \delta$. Combining these with Lemma 3.2 we can conclude that $w_{1}(\xi)$ is a supersolution of (3.1). Analogously, $w_{2}(\xi)$ is a subsolution of (3.1). Hence from the choice of $u$ and the fact that every supersolution dominates every subsolution, we have in $\Omega$ that

$$
w_{2}(\xi) \leq u(\xi) \leq w_{1}(\xi)
$$

and then

$$
|u(\xi)-\varphi(\zeta)| \leq \varepsilon+k w(\xi) .
$$

Since $w(\xi) \rightarrow 0$ as $\xi \rightarrow \zeta$, we obtain $u(\xi) \rightarrow \phi(\zeta)$ as $\xi \rightarrow \zeta$.

Remark 3.12. Let $f \in C^{\infty}(\Omega) \cap C(\bar{\Omega})$ and $u$ be the solution of

$$
\begin{cases}L u-\lambda(\xi) u=f, & \text { in } \Omega \\ u=0, & \text { on } \partial \Omega\end{cases}
$$

Elementary calculations show that $-\frac{1}{\min _{\xi \in \Omega} \lambda(\xi)}\|f\|_{L^{\infty}(\Omega)}$ and $\frac{1}{\min _{\xi \in \Omega} \lambda(\xi)}\|f\|_{L^{\infty}(\Omega)}$ are a subsolution and a supersolution of (3.5) respectively. Thus, $\|u\|_{L^{\infty}(\Omega)} \leq \frac{1}{\min _{\xi \in \Omega} \lambda(\xi)}\|f\|_{L^{\infty}(\Omega)}$. It provides a $L^{\infty}$ estimate for the solution of (3.5).

Theorem 3.13. Set $\phi \in C(\partial \Omega)$ and $f \in C(\bar{\Omega})$. Then there exists a unique solution $u \in C(\bar{\Omega})$ to (3.1) in the sense of distribution.

Proof. Take a sequence $f_{n}(\xi) \in C^{\infty}(\Omega) \cap C(\bar{\Omega}), n=1,2, \ldots$, so that $\left\{f_{n}(\xi)\right\}$ converges uniformly to $f$ in $\Omega$. Denote by $u_{n}$ the corresponding solution of the Dirichlet problem

$$
\begin{cases}L v-\lambda(\xi) v=f_{n}(\xi), & \text { in } \Omega \\ u=\varphi, & \text { on } \partial \Omega\end{cases}
$$

We obtain, in view of Remark 3.12,

$$
\left\|u_{n}-u_{m}\right\|_{L^{\infty}(\Omega)} \leq \frac{1}{\min _{\xi \in \Omega} \lambda(\xi)}\left\|f_{n}-f_{m}\right\|_{L^{\infty}(\Omega)} .
$$


In conclusion, $\left\{u_{n}\right\}$ converges uniformly to a continuous function $u$ which is the required solution.

\section{The monotone iteration scheme for semilinear equation}

Let $\Omega$ be a bounded open domain in a Carnot group G. Consider Dirichlet problem (1.1), where $f(\xi, u)$ is a smooth function of $\xi$ and $u, \phi \in C(\partial \Omega)$. A function $\mu \in C(\bar{\Omega})$ is called a supersolution of (1.1) if it satisfies

$$
\begin{cases}L \mu+f(\xi, \mu) \leq 0, & \text { in } \Omega \\ \mu(\xi) \geq \varphi(\xi), & \text { on } \partial \Omega\end{cases}
$$

Analogously, a function $v \in C(\bar{\Omega})$ is called a subsolution of (1.1) if it satisfies

$$
\begin{cases}L v+f(\xi, v) \geq 0, & \text { in } \Omega \\ v(\xi) \leq \varphi(\xi), & \text { on } \partial \Omega\end{cases}
$$

The above inequalities are both in the sense of distribution. Here, a function $T \geq 0$ means that for any positive test function $\psi$, we have $T \psi \geq 0$. In the following we are ready to construct a smooth solution of (1.1) commencing with a subsolution and a supersolution in $S^{1,2}(\Omega, l o c)$ by the monotone iteration scheme. We first prove a maximum principle.

Lemma 4.1. Assume that $u \in S^{1,2}(\Omega) \cap C(\bar{\Omega})$ satisfies

$$
L u-\lambda(\xi) u \geq 0,
$$

where $\lambda(\xi) \in C(\bar{\Omega})$ and $\lambda(\xi)>0$. If $u \leq 0$ on $\partial \Omega$, then $\sup _{\xi \in \Omega} u(\xi) \leq 0$.

Proof. Suppose that the conclusion fails. Since $u$ is continuous on $\bar{\Omega}$, there exists a point $\xi_{0} \in \Omega$ such that $u\left(\xi_{0}\right)>0$. Fix $\varepsilon>0$ so small that $u\left(\xi_{0}\right)-\varepsilon>0$. Consequently, the function $u_{\varepsilon}:=\max \{u-\varepsilon, 0\}$ is non-negative and has compact support in $\Omega$ as $u \leq 0$ on $\partial \Omega$. By the distribution meaning of solutions, we get

$$
\int_{\Omega} X u \cdot X u_{\varepsilon} d G=\int_{\Omega}-u_{\varepsilon} L u d G \leq \int_{\Omega}-\lambda(\xi) u u_{\varepsilon} d G \leq 0 .
$$

When $u_{\varepsilon}>0$, it follows $X u_{\varepsilon}=X u$ and $X u$ is not identically zero. In fact, if $X u \equiv 0$, then $u \equiv u\left(\xi_{0}\right)>0$ in $\Omega$ which contradicts the assumption that $u \leq 0$ on $\partial \Omega$. Consequently the left hand side of (4.1) is positive, a contradiction. This completes the proof of the lemma. $\square$

Theorem 4.2. Let $\Omega$ be as in Lemma 3.10. Let $f \in C^{\infty}(G \times(a, b))$ and $\phi \in C(\partial \Omega)$. Suppose that $\mu$ and $v$ are, respectively, a supersolution and a subsolution of (1.1) with $\mu, v \in S^{1,2}(\Omega, l o c) \cap C(\bar{\Omega}), v \leq \mu$, and $a<\min v<\max \mu<b$. Then there exists a solution $u \in C^{\infty}(\Omega) \cap C(\bar{\Omega})$ of (1.1) satisfying $v \leq u \leq \mu$.

Proof. Take $K>0$ such that

$$
\frac{\partial f}{\partial u}+K^{2}>0
$$

on $\bar{\Omega} \times[\min v, \max \mu]$. Let $v=T u$ denote the unique solution in $C(\bar{\Omega})$ of the Dirichlet problem (see Theorem 3.11) 


$$
\begin{cases}\left(L-K^{2}\right) v=-\left[f(\xi, u)+K^{2} u\right], & \text { in } \Omega \\ v(\xi)=\varphi(\xi), & \text { on } \partial \Omega\end{cases}
$$

We claim that the nonlinear transformation $T$ is monotone. To establish this we set $u_{1}<u_{2}$ and notice that

$$
\begin{aligned}
& \left(L-K^{2}\right) T u_{1}=-\left[f\left(\xi, u_{1}\right)+K^{2} u_{1}\right] \\
& \left(L-K^{2}\right) T u_{2}=-\left[f\left(\xi, u_{2}\right)+K^{2} u_{2}\right]
\end{aligned}
$$

and $T u_{1}=T u_{2}=\phi$ on $\partial \Omega$. Letting $w=T u_{1}-T u_{2}$, we can obtain

$$
\left(L-K^{2}\right) w=-\left[f\left(\xi, u_{1}\right)-f\left(\xi, u_{2}\right)+K^{2}\left(u_{1}-u_{2}\right)\right]
$$

and $w=0$ on $\partial \Omega$. As $f(\xi, u)+K^{2} u$ is increasing in $u$ by (4.2), it yields $\left(L-K^{2}\right) w \geq 0$. From

$$
L w=K^{2} w-\left[f\left(\xi, u_{1}\right)-f\left(\xi, u_{2}\right)+K^{2}\left(u_{1}-u_{2}\right)\right],
$$

we get $w S^{2,2}(\Omega, l o c)$ by $L w \in L^{2}(\Omega)$ and Proposition 2.1-(i). It follows that $w \leq 0$ in $\Omega$ by applying Lemma 4.1 , therefore, $T u_{1} \leq T u_{2}$ and $T$ is monotone. We now begin the iteration scheme.

Let $u_{1}=T \mu$. As

$$
\left(L-K^{2}\right) u_{1}=-\left[f(\xi, \mu)+K^{2} \mu\right]
$$

and $u_{1}=\phi$ on $\partial \Omega$, we get by a trivial calculation that

$$
\left(L-K^{2}\right)\left(u_{1}-\mu\right)=-[L \mu+f(\xi, \mu)] \geq 0
$$

and $u_{1}-\mu \leq 0$ on $\partial \Omega$. Arguing as in the previous gives $u_{1} \leq \mu$ in $\Omega$.

Define $u_{n+1}=T u_{n}$. The monotoneity of $T$ yields

$$
\mu \geq u_{1} \geq u_{2} \geq \cdots .
$$

Analogously, starting from $v$, we obtain a nondecreasing sequence

$$
v \leq v_{1} \leq v_{2} \leq \cdots,
$$

where $v_{1}=T v, v_{n+1}=T v_{n}$. Moreover, $v \leq \mu$ implies $v_{1}=T v \leq T \mu=u_{1}$ and, therefore, $v_{n} \leq u_{n}$ for each $n \in \mathbb{N}$. Thus

$$
v \leq v_{1} \leq v_{2} \leq \cdots \leq v_{n} \leq u_{n} \leq \cdots \leq u_{2} \leq u_{1} \leq \mu,
$$

so that the limit $u=\lim _{n \rightarrow \infty} u_{n}$ is well defined in $\bar{\Omega}$. Recall that

$$
\left(L-K^{2}\right) u_{n+1}=-\left[f\left(\xi, u_{n}\right)+K^{2} u_{n}\right] .
$$

The dominated convergence theorem shows that

$$
L u+f(\xi, u)=0
$$

in the distributional sense. According to Proposition 2.1-(i) and the fact that $f(\xi, u) \in$ $L^{p}(\Omega)$ for $1<p<+\infty$ one has $u \in S^{2, p}(\Omega, l o c)$. Iterating the process, we get $u \in S^{k, p}(\Omega$, loc) for $k \geq 0$. Let $\psi \in C_{0}^{\infty}(\Omega)$. The definition in Section 2 gives $\psi u \in S^{k, p}$. 
Furthermore, we obtain $u \in C^{\infty}(\Omega)$ in view of Proposition 2.1-(ii). Combining this with (4.3) we have $u \in C^{\infty}(\Omega) \cap C(\bar{\Omega})$ which is the desired solution.

We assume henceforth that $G$ is of Heisenberg type. Such group was introduced by Kaplan [14] and has been subsequently studied by several authors, see $[4,11,13]$ and the references therein.

Let $G$ be a Carnot group of step two whose Lie algebra $\tilde{g}=V_{1} \oplus V_{2}$. Consider the map $J: V_{2} \rightarrow \operatorname{End}\left(V_{1}\right)$ defined by

$$
\left\langle J\left(\xi_{2}\right) \xi_{1}^{\prime}, \xi_{1}^{\prime \prime}\right\rangle=\left\langle\xi_{2},\left[\xi_{1}^{\prime}, \xi_{1}^{\prime \prime}\right]\right\rangle, \text { for } \xi_{1}^{\prime}, \xi_{1}^{\prime \prime} \in V_{1} \text { and } \xi_{2} \in V_{2} .
$$

$G$ is said of Heisenberg type if for every $\xi_{2} \in V_{2}$, with $\left|\xi_{2}\right|=1$, the map $J\left(\xi_{2}\right): V_{1} \rightarrow$ $V_{1}$ is orthogonal.

In the case of the Heisenberg type groups, the gauge balls coincide with the level sets of the fundamental solution (that is a radial function in this class of groups, see [14]), and the balls $B_{G}(e, R)$ invade $G$ as $R$ tends to $+\infty$ since the vector fields on $G$ satisfy the Hörmander rank condition. Thus, we get the following existence theorem in the whole space $G$ by making use of Theorem 4.2 and the result in [4] that the gauge balls in H-type group satisfy the outer sphere condition.

Theorem 4.3. Let $G$ be a group of Heisenberg type. Let $u_{-}(\xi), u_{+}(\xi) S^{1,2}(G, l o c) \cap C(G)$ be respectively a subsolution and a supersolution of the problem

$$
L u+f(\xi, u)=0,
$$

where $f \in C^{\infty}(G \times(a, b))$ and $a<u_{-}(\xi) \leq u_{+}(\xi)<b$. Then there exists a solution $u \in$ $C^{\infty}(G)$ of (4.4) satisfying

$$
u_{-} \leq u \leq u_{+}
$$

in $G$.

Proof. Let $u_{0}=u_{+}$, set $B_{G}(e, m)$ be the gauge ball of radius $m$ centered at identity $e$. We construct $u_{m}$ inductively in the following manner. Let $v_{m}$ be the solution of the Dirichlet problem

$$
\begin{cases}L v+f(\xi, v)=0, & \text { in } B_{G}(e, m) \\ v(\xi)=u_{+}(\xi), & \text { on } \partial B_{G}(e, m)\end{cases}
$$

obtained by means of Theorem 4.2 using $u_{-}$and $u_{m-1}$, respectively, as a subsolution and a supersolution.

Define

$$
u_{m}(\xi)=\left\{\begin{array}{l}
v_{m}(\xi), \xi \in B_{G}(e, m) \\
u_{+}(\xi), \xi \notin B_{G}(e, m) .
\end{array}\right.
$$

Obviously, $u_{-} \leq u_{m} \leq u_{m-1}$. We need to prove that $u_{m}$ is a supersolution of (4.4). To see this, take a positive test function $\psi(\xi) \in C_{0}^{\infty}(G)$. From the divergence theorem, we obtain

$$
\begin{aligned}
\int_{B_{G}(e, m)} v_{m} L \psi d G & =\int_{B_{G}(e, m)} \psi L v_{m} d G+\int_{\partial B_{G}(e, m)} v_{m}\langle A \nabla \psi, \vec{n}\rangle d S \\
& -\int_{\partial B_{G}(e, m)} \psi\left\langle A \nabla v_{m}, \vec{n}\right\rangle d S
\end{aligned}
$$


and

$$
\begin{aligned}
\int_{G \backslash B_{G}(e, m)} u_{+} L \psi d G & =\int_{G \backslash B_{G}(e, m)} \psi L u_{+} d G+\int_{\partial B_{G}(e, m)} \psi\left\langle A \nabla u_{+}, \vec{n}\right\rangle d S \\
& -\int_{\partial B_{G}(e, m)} u_{+}\langle A \nabla \psi, \vec{n}\rangle d S .
\end{aligned}
$$

The above two identities give

$$
\begin{aligned}
\int_{G} u_{m} L \psi d G= & \int_{B_{G}(e, m)} \psi L v_{m} d G+\int_{G \backslash B_{G}(e, m)} \psi L u_{+} d G \\
& +\int_{\partial B_{G}(e, m)} \psi\left\langle A \nabla\left(u_{+}-v_{m}\right), \vec{n}\right\rangle d S
\end{aligned}
$$

where $\vec{n}$ denotes the outerward normal to $\partial B_{G}(e, m)$, and $A$ is a fixed positive semidefinite matrix (see $[4,13])$. Therefore, we may restrict ourselves to the case in which $\left\langle A \nabla\left(u_{+}-v_{m}\right), \vec{n}\right\rangle$ represents the derivative of $u_{+}-v_{m}$ in an outward direction with respect to $\partial B_{G}(e, m)$. Moreover, since $u_{+}-v_{m} \geq 0$ in $B_{G}(e, m)$ and $u_{+}-v_{m}=0$ on $\partial B_{G}$ $(e, m)$, it follows

$$
\psi\left\langle A \nabla\left(u_{+}-v_{m}\right), \vec{n}\right\rangle \leq 0 \quad \text { for } \quad \xi \in \partial B_{G}(e, m) .
$$

Substitution in (4.5) gives

$$
\begin{aligned}
\int_{G} u_{m} L \psi d G & \leq-\int_{B_{G}(e, m)} \psi f\left(\xi, v_{m}\right) d G-\int_{G \backslash B_{G}(e, m)} \psi f\left(\xi, u_{+}\right) d G \\
& =-\int_{G} \psi f\left(\xi, u_{m}\right) d G
\end{aligned}
$$

This implies that $u_{m}$ is a supersolution, and we can restart the monotone iteration scheme on $B_{G}(e, m+1)$.

In this way we obtain iteratively a sequence of supersolutions $\left\{u_{m}\right\}$ satisfying the following properties:

(i) $\left\{u_{m}\right\}$ is nonincreasing, and $u_{-} \leq u_{m} \leq u_{+}$;

(ii) Every $u_{m}$ satisfies $L u_{m}+f\left(\xi, u_{m}\right)=0$ in $B_{G}(e, m)$.

Set $u(\xi)=\lim _{m \rightarrow \infty} u_{m}(\xi)$. We observe that $\left\{u_{m}\right\}$ is a sequence of solutions of (4.4) on any $B_{G}(e, k)$ for $m \geq k$. It follows that $u$ is a solution on $B_{G}(e, k)$. Arguing as in Theorem 4.2 we know $u \in C^{\infty}\left(B_{G}(e, k)\right)$. The arbitrariness of $k$ implies $u \in C^{\infty}(G)$. Therefore, it holds that $u$ is the required solution of (4.4).

\section{Acknowledgements}

We would like to thank Pengcheng Niu for research assistance and the two anonymous referees for very constructive comments. Zixia Yuan thanks the Mathematical Tianyuan Youth Foundation of China (No. 11026082) for financial support.

Author details

${ }^{1}$ School of Mathematical Science, University of Electronic Science and Technology of China, Chengdu 611731, China

${ }^{2}$ Department of Mathematics, Henan Institute of Science and Technology, Xinxiang 453003, China 


\section{Competing interests}

The authors declare that they have no competing interests.

Received: 7 December 2011 Accepted: 12 June 2012 Published: 12 June 2012

\section{References}

1. Folland, GB: Subelliptic estimates and function spaces on nilpotent Lie groups. Ark Math. 13(1-2), 161-207 (1975). doi:10.1007/BF02386204

2. Bony, J: Principe du maximum, inégalité de Harnack et unicité du problème de Cauchy pour les opérateurs elliptiques dégénérés. Ann Inst Fourier (Grenoble). 19(1), 277-304 (1969). doi:10.5802/aif.319

3. Negrini, P, Scornazzani, V: Wiener criterion for a class of degenerate elliptic operators. J Diff Eq. 66(2), 151-164 (1987). doi:10.1016/0022-0396(87)90029-5

4. Capogna, L, Garofalo, N, Nhieu, DM: Properties of harmonic measures in the Dirichlet problem for nilpotent Lie groups of Heisenberg type. Am J Math. 124(2), 273-306 (2002). doi:10.1353/ajm.2002.0010

5. Perron, O: Eine neue Behandlung der ersten Randwertaufgabe für $\Delta u=0$. Math Z. 18(1), 42-54 (1923). doi:10.1007/ BF01192395

6. Gilbarg, D, Trudinger, NS: Elliptic Partial Differential Equations of Second Order. Springer, Berlin, 2 (1983)

7. Amann, H: Supersolution, monotone iteration and stability. J Diff Eq. 21(2), 363-377 (1976). doi:10.1016/0022-0396(76) 90126-1

8. Deng, YH, Chen, G, Ni, WM, Zhou, JX: Boundary element monotone iteration scheme for semilinear elliptic partial di erential equations. Math Comput. 65(215), 943-982 (1996). doi:10.1090/S0025-5718-96-00743-0

9. Brandolini, L, Rigoli, M, Setti, AG: Positive solutions of Yamabe-type equations on the Heisenberg group. Duke Math J. 91(2), 241-296 (1998). doi:10.1215/50012-7094-98-09112-8

10. Gaveau, B: Principe de moindre action, propagation de la chaleur et estimées sous elliptiques sur certains groupes nilpotents. Acta Math. 139(1), 95-153 (1977). doi:10.1007/BF02392235

11. Garofalo, N, Vassilev, D: Regularity near the characteristic set in the non-linear Dirichlet problem and conformal geometry of sub-Laplacians on Carnot groups. Math Ann. 318(3), 453-516 (2000). doi:10.1007/s002080000127

12. Han, YZ, Luo, XB, Niu, PC: Liouville type theorems of semilinear equations with square sum of vector fields. J Part Diff Eq. 18(2), 149-153 (2005)

13. Garofalo, N, Vassilev, D: Symmetry properties of positive entire solutions of Yamabe-type equations on groups of Heisenberg type. Duke Math J. 106(3), 411-448 (2001). doi:10.1215/S0012-7094-01-10631-5

14. Kaplan, A: Fundamental solutions for a class of hypoelliptic PDE generated by composition of quadratic forms. Trans Am Math Soc. 258(1), 147-153 (1980). doi:10.1090/S0002-9947-1980-0554324-X

doi:10.1186/1029-242X-2012-136

Cite this article as: Yuan and Yuan: Dirichlet problems for linear and semilinear sub-Laplace equations on Carnot groups. Journal of Inequalities and Applications 2012 2012:136.

\section{Submit your manuscript to a SpringerOpen ${ }^{\odot}$ journal and benefit from:}

- Convenient online submission

- Rigorous peer review

- Immediate publication on acceptance

- Open access: articles freely available online

- High visibility within the field

- Retaining the copyright to your article

Submit your next manuscript at $\gg$ springeropen.com 